\title{
DREAM \& TEAM: A Tool and a Notation Supporting Exploration of Options and Traceability of Choices for Safety Critical Interactive Systems
}

\author{
Xavier Lacaze and Philippe Palanque \\ LIIHS - IRIT, Université Paul Sabatier \\ 118 route de Narbonne, 31062 Toulouse Cedex 4 \\ \{lacaze, palanque\} @irit.fr \\ http://liihs.irit.fr/palanque
}

\begin{abstract}
Justification of choices made throughout the design process of systems is a recurrent desire and quite often a formal request from certification authorities in the safety critical domain. However, even though some work has already been done in the early phases of the development processes, justifying choices in the later phases such as detailed design or implementation remain a cumbersome activity left (without any support) in the hands of the developers. This paper presents a notation called TEAM (Traceability, Exploration and Analysis Model) and its associated tool called DREAM (Design Rationale Environment for Argumentation and Modelling). The paper presents first the notation and its specificities with respect to other Design Rationale notations. Both the notation and the tools are presented on a case study showing how they can support design of interaction techniques for Air Traffic Control workstations. We also present the rationale that we have gathered while designing the graphical representation of the notation.
\end{abstract}

\section{Introduction}

Traceability of choices is a critical aspect of the development processes in the field of safety critical systems. Some standards, and especially in the field of safety critical systems, such as DO 178 B [21]) defines the guidelines for development of aviation software. This standard explicitly requires the use of methods and techniques for systematically exploring design options and for increasing traceability of design decisions. DO $178 \mathrm{~B}$ is a document describing a design process, however, even though it is widely used in the aeronautical domain, the design rationale part remains superficially addressed without any guidance for the designers or developer on how to reach the objectives. Similarly, the ESARR (Eurocontrol Safety Regulatory Requirement) on Software in Air Traffic Management Systems [6] explicitly requires traceability to be addressed in respect of all software requirements (pp. 11 edition 0.2).

While traceability has been a recurring concern in the field of HCI since the late 80's (reaching a climax in 1996 with the book survey [17]) there is still no mature enough notation and tool to engineer traceability i.e. to support the exploration of options and the traceability of choices made throughout the development process.

This paper presents such a notation called TEAM and its associated tool called DREAM. The notation extends QOC notation [15] in a way to be especially suited for 
the traceability of interactive systems. The tool supports the edition and the exploitation of models. Visualization techniques have been embedded to support specifically the tasks associated to models exploitation.

Section 2 describes previous work in the field of design rationale and more precisely work dealing with interactive systems. Last part of this section introduces some criteria used to compare this previous work and to rationalize the need for additional work in order to support interactive system designers' activities. Section 3 presents the DREAM-TEAM approach i.e. both the notation and the tool. The description of the notation TEAM is based on QOC and has an emphasis on extensions that have been proposed and the rationale for their addition. Then the DREAM tool is rapidly presented. The tool is available for download on the web at the following address: http://liihs.irit.fr/dream. Last section (section 4) presents the actual use of DREAMTEAM approach on a case study in the filed of interactive Air Traffic Management workstations. This case study applies the approach to interaction technique design for clearances' (orders given to the pilots in the approach phase) input.

\section{Related Work}

This section presents related work on design rationale. It positions the work described in the paper with respect to the current state of the art in the field. It is structured around two aspects: notations for modelling and storing information and tools that support the edition and retrieval of information stored in models. In order to fit the number of pages limitations we have gathered screenshots of the tools in one single figure: Fig. 1. These tools are presented in detail in the following sections.

\subsection{IBIS - gIBIS}

gIBIS [4] (Graphical IBIS) is a tool supporting design activities. Main objective of gIBIS is to capture design rationale emerging during these activities. gIBIS supports IBIS (Issue-Based Information System) notation [10]. A snapshot is provided on area 2 (bottom left corner) of Fig. 1.

IBIS notation is the first notation that explicitly relates design activities and design rationale. IBIS main goal is to capture decisions that are made and so with an historical perspective (i.e. how and why designers have taken this decision). IBIS breaks down design into Issues. An Issue could be related to Answer, Statements, or Positions. These three elements are linked with one or more Arguments (positive or negative). A network, called issue map, represents dependencies between Issues. Issues are connected together by four kinds of dependencies: more general than, similar to, temporal successor of, logical successor of. The main drawback of this notation lays in the arguments. Arguments support or deny one solution. Each solution has pro and cons arguments, but there is no way to compare solutions. gIBIS does not provide any support for handling large diagrams such as nesting, duplication of issues, ... This is not a problem when small diagrams are edited, but when it come to real size applications, not supporting scalability may lead to inconsistent diagram.

gIBIS extends IBIS notation by adding two kinds of nodes in order to improve flexibility: 
(1) external, allows integrating other electronic documents (pictures, videos, mail, text etc.);

(2) other, this node include all thinks users can not associate to other nodes. And two links are added, one for generalised a position and one in order to specialise a position.

gIBIS users are supposed to focus on three points: to capture design history, to support several kinds of conversation media (mail, news, etc.), to search information into diagrams, and navigate on diagrams. Fig. 1 (area 2) provides a snapshot of the gIBIS environment. On the left part, a diagram is displayed with both detailed and global view. Top right corner displays textual representation of the diagram. The set of buttons provides functions for diagram edition (add node, add link, delete, etc.) while the right corner displays additional textual information about currently selected node.
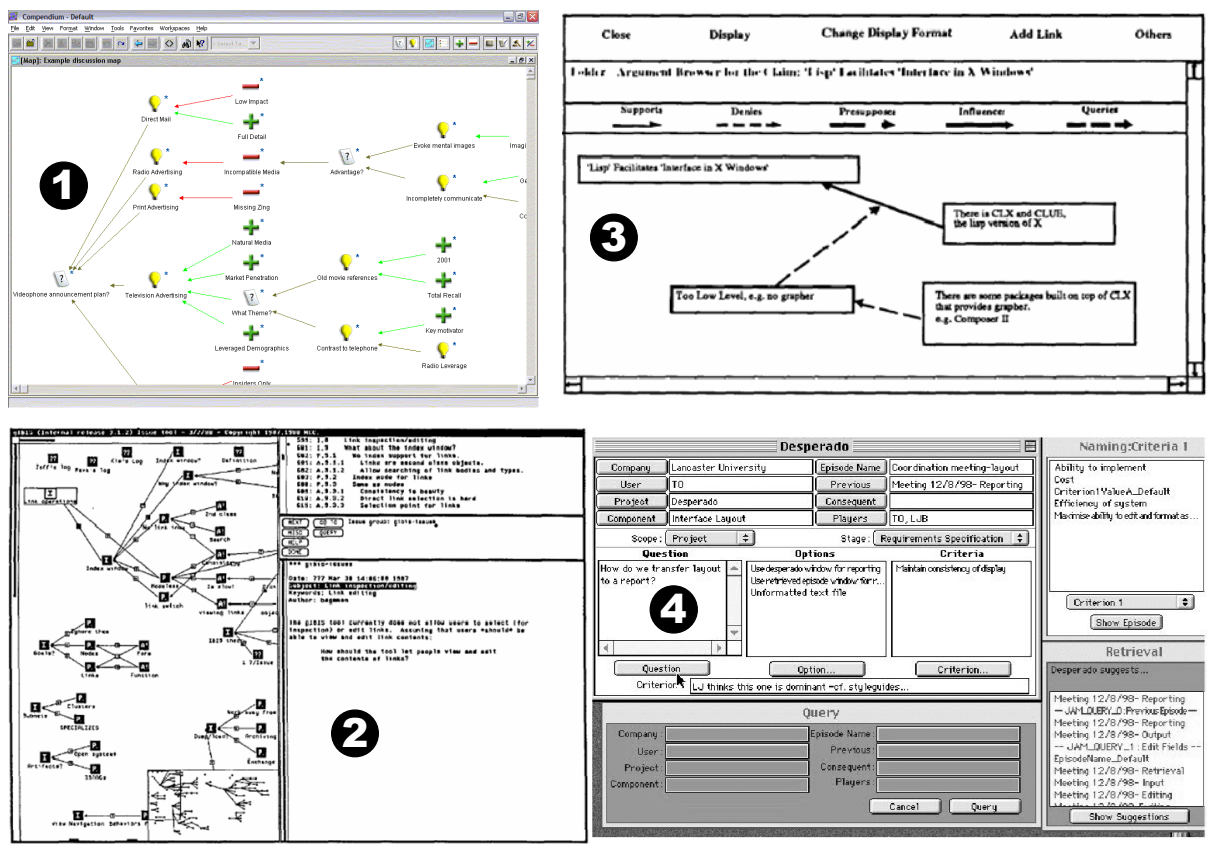

Fig. 1. Screenshot of four environments dedicated to design rationale

gIBIS was evaluated by a one year study [5]. From this study it is claimed that most users understood usefulness of design rationale thanks to the tool. This was not the case during the learning stage of the notation without tool support. However, the study has also shown that the tool required sequential editing of diagrams i.e. first issue then position and argument. It is not allowed, for instance, to enter arguments without having first edited issue and position. However, due to this constraint, gIBIS diagrams are always syntactically correct. 


\subsection{IBIS - Compendium}

Compendium [3] is based on IBIS notation too. Compendium is a follow up of QuestMap and improves it by allowing the integration of Excel ${ }^{\mathrm{TM}}$ and Word ${ }^{\mathrm{TM}}$ files into diagrams. Compendium features a graphical interface and has been used in several projects both academic and industrial. Authors are not satisfied about the tool and they have shown that users did not use the tool in the expected/right way. They used it to keep history of ideas, solutions, and as shared memory. They did not use it as a tool to capture rationale during the design phases as they were supposed to.

A snapshot of the environment is proposed on Fig. 1 (area 1). Each node is associated with an icon. Issues are displayed as a question mark, positions as a light bulb, positive arguments as green plus, and negative arguments as red minus. Users have to be logged on the system in order to use it. This feature allows Compendium to store data about who made modification and when they were made. In its current state, the tool only stores the date of creation and of last modification of nodes.

No global visualisation of the diagram is available which makes cumbersome the activity of working with large diagrams. Lack of global visualisation of the diagram slows down the navigation. Compendium allows users to provide more information about a node by adding textual information but there is no mean to access this information by searching for instance and thus this information remains at a too low level to be usable. The number of appearances of a given node throughout the diagram is provided by the system, but there is no support for locating the other instances of a given node. Additional feature allows exporting diagram into html, textual or picture file that could easily be included in a documentation report. This notion of documentation report is of prime importance when dealing with safety critical systems as paper documents can be used as legal documents.

\subsection{DRL - SIBYL}

The aim of SIBYL [12] is to support group work during decision making phases and is based on DRL notation (Decision Representation Language) [14]. DRL aims at capturing decision made during the design process. DRL is made of edges and nodes and thus offers a graphical representation for diagrams. Each node is considered as an object of the following type: alternative, goal (i.e. the decision problem), question, group, viewpoint, procedure, status (decided or not) and claim. Edges can only connect two objects and are of the following types: achieves (alternative, goal); supports (claim, claim); denies (claim, claim); presupposes (claim, claim); is a sub goal of (goal, goal); answers (claim, question); is an answering procedure for (procedure, question); is a result of (claim, procedure); tradeoffs(object, object, attribute); is a kind of (object, object); suggests (object, object).

DRL notation allows users to split a design problem into one or more sub problems. As for IBIS notation, each option is evaluated independently from the others. The resulting diagram is highly structured. Scalability is a also a main issue for this notation as diagrams grow up rapidly and feature a lot of edges crossings and this makes DRL a not easy to use notation (both in terms of reading and producing diagrams). 
SIBYL (see area 3 of Fig. 1) supports DRL. SIBYL was built following Potts and Bruns model [20], [13]. This model can be seen as a set of guideline for building interactive system supporting a design rationale notation. SIBYL is implemented with a database where information is stored in and can be retrieved from. Even though a Graphical representation is provided there is no support for global visualisation.

\subsection{QOC - Desperado}

Desperado [19] is based on QOC notation. As the work presented in this paper builds upon QOC too, we present this notation in more detail than the previous ones. In this section we first introduce the notation and then we describe Desperado tool. Other tools have been proposed to support the edition of QOC diagrams as for instance [22] but Desperado is, from our point of view the more mature.

QOC (Questions, Options, Criteria), is a semi formal notation (see Fig. 2) meant to be is easy to use and to understand. Each actor, involved in the design process, could understand and use the notation whatever their background is.

A typical QOC diagram can be break down into three columns and links between items of columns. Each row represents a notation's item: questions, options, and criteria. For one question, several options are associated. Option represents a design solution for the question. An option is evaluated by several criteria, each criterion supports (strong link) or denies (doted line link) the option. A criterion can be used to evaluate several options and thus may appear several times in the diagram. Criteria permit to model qualitative comparison between options. When choices are made, designers have to select (according to criteria for instance) the option. The selected option (is framed (see for instance option3 in Fig. 2) and usually the selected option is the one that satisfies the larger number of criteria. In QOC, an option may lead to another question (as for instance question 2 in Fig. 2) thus explicitly showing links between diagrams. In addition, arguments can be attached to link between options and criteria in order to describe with further detail, either the content or the underlying rationale for the value assigned to the link. The explicit handling of criteria is one of the main advantages of QOC with respect to other design rationale notations.

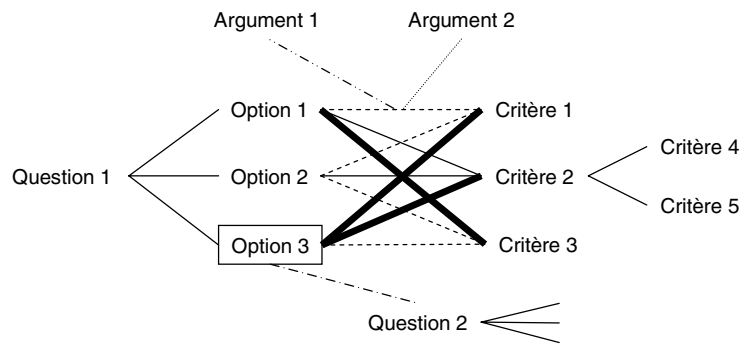

Fig. 2. An example of a QOC diagram

A snapshot of Desperado environment is provided in area 4 of Fig. 1. Desperado was built for storing and reusing design rationale data. Desperado is related with a data base storing all the information. Edition of models is only sequential and is done by means of form filling following four steps: First step (set up), users have to fill in 
context related information (question description, user name, design step, people related, etc.). The tool helps users by providing data from the database. In the second step, users fill in options, criteria, and provide an impact value of criteria to options. Some values may be prompted by Desperado according to the other values for the other options in the diagram. At step three, users index relevant electronic documents. Last step allows users to create new questions related to the one under consideration and thus refine the model into a sub-model. Users can also search (using keywords request), and modify previously edited diagrams.

Editing phase is constrained due to the imposed sequence data input. The tool does not make it possible to store pending questions, questions not yet resolved (after a question the user must edit options). There is no global representation of data, making it hard to handle large models event though this is a typical size for DR diagrams.

\subsection{Comparative Analysis}

In this section, we provide a comparative analysis of the notations and their environments tool plus notation. Comparison is made by criteria. Notations (first column in Table 1) are evaluated with respect to arguments (possibility to plug argument in the diagram), valuations (level of valuation), criterion and hierarchical (possibility to build nested diagram). The relation to criterion is ticked if the corresponding notation allows for a given criterion to be related to several options in order to provide a comparative analysis of options. Tools (second column in Table 1) are compared with the following criteria (last column in Table 1): database (link with a database or not), graphical displays (included direct manipulation), links with other electronic documents, and the implementation of specific features supporting the edition phase (such as undo/redo, etc.).

Table 1. Summary of evaluation of design rationale notations and tools

\begin{tabular}{|c|c|c|c|c|c|c|c|c|c|}
\hline \multirow{3}{*}{ IBIS } & \multirow{3}{*}{$\begin{array}{c}\text { gIBIS } \\
\text { Compendium }\end{array}$} & \multicolumn{4}{|c|}{ Notations } & \multicolumn{4}{|c|}{ Tools } \\
\hline & & 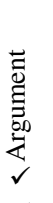 & 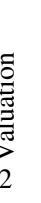 & 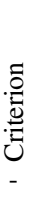 & 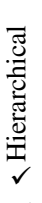 & 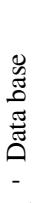 & 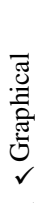 & 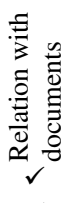 & 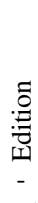 \\
\hline & & $\checkmark$ & 2 & - & $\checkmark$ & $\checkmark$ & $\checkmark$ & $\checkmark$ & $\checkmark$ \\
\hline DRL & SIBYL & $\checkmark$ & 2 & - & - & - & - & - & - \\
\hline QOC & Desperado & $\checkmark$ & 3 & $\checkmark$ & $\checkmark$ & $\checkmark$ & - & - & - \\
\hline
\end{tabular}

Evaluation of notations and tools, as shown in Table 1 gives two main information about QOC and IBIS notations. QOC is the only notation meeting the criterion attribute. QOC also offers three levels of valuation between criteria and options. QOC satisfies all criteria we decided to evaluate but its related tool Desperado satisfies only one criterion. Concerning IBIS, gIBIS and Compendium are useful tools (they allow the edition of diagrams and information storage) but they embed the limitations of IBIS notation. Besides, none of the notations and tools defines clear relationships with 
Human Computer Interaction concerns and they do not provide any support for dealing with specific information for the design of interactive system.

Following this comparative study and the requirements extracted from our projects in the field of safety critical interactive systems, we purpose hereafter to extend QOC notation in order to embed HCI components and empower the notation. We also designed a tool supporting this notation meeting most of the criteria presented above.

\section{DREAM and TEAM Approach}

TEAM (Traceability, Exploration and Analysis Mode)) \& DREAM (Design Rationale Environment for Argumentation and Modelling) are a new approach dedicated to the support of systematic exploration of option and traceability of decision that are made throughout the development of safety critical interactive systems. As stated above TEAM is based on QOC notation [15], so, building on section 2, we first present all the extensions we designed from standard QOC and then how DREAM has been designed to support the edition and manipulation of TEAM diagrams.

\subsection{Extensions}

Currently we have embedded four extensions to 'standard' QOC diagrams in order to support the specific needs for interactive systems engineering: task model and scenarios, ergonomic criteria and usability factors, weights to criteria and factors and connection to software architecture models for interactive systems.

Tasks models extension [11] aims at integrating task models in QOC diagrams in order to be able (through scenarios extracted from the task models) to assess respective performance of the various options under consideration. As task analysis and modelling is at the core of User Centred Design [18], integration of task models must be embedded in the rationalisation process. Besides, the extraction of scenarios from task models provides precise and concrete information for assessing the relative efficiency and usability of different design options. In TEAM, task models are connected to options thus allowing representing the fact that an option is able to support the performance of a given task by the user.

Ergonomic criteria and usability factors extension has been introduced by Farenc and Palanque [7]. In the original QOC there is no way to store and thus to argue with respect to user and stakeholders requirements. However, it is clear that in User Centred Development, users play an important role in the decisions made. In this extensions user requirements are expressed as a set of factors. The factors correspond to high-level requirements such as learnability, safety, usability, etc., and some criteria may increase or decrease the satisfaction of a factor. A factor can be connected to one or several criterion. The early identification of factors has been based on McCall's classification [16] that is widely used in software engineering. The elements of the classification are the following: (1) quality factors (requirements expressed by the clients and/or users); (2) quality criteria (that can be measured for a given option according to a given scenario); (3) metrics: allow the actual valuation of a criterion. 
According to studies done by Shum [23] QOC notation can transcribe exactly what users' want but a remaining issue is that criteria are at the same level i.e. it is not possible to say that a given criterion is more important than another one. Applying the notation in the field of safety critical interactive systems we have seen that such weighting of criteria is required as this is very often mentioned when decisions are taken. For this reason, we propose to associate to criterion and factor a weight describing their relative importance for stakeholders and users. The weight as defined in TEAM can have up to five valuations: starting from 1 (important) to 5 (optional). For the same reasons a weight is also associated to each criterion but for a given option. Thus in a given diagram a given criterion might be weighted as important while being weighted as optional in another diagram. Similarly, weights are associated to factors. The actual weight of a factor has an impact on the weight of the related criteria.

Arch model [0] is an extension of Seeheim model [8]. Arch breaks down an interactive system into five components: the domain specific component, the domain adaptor component, the dialogue component, the logical level interaction component, and the interaction toolkit component. Arch is both generic and precise enough to provide a framework for structuring design rationale diagrams. Our extension proposal is to relate each question to a component of the Arch model. As we are dealing with interactive systems design, we have decided to merge the domain specific component and the domain adaptor component into one called functional core. As the argumentation is often targeted to presentation (in the broad sense) and not towards one of the two presentation components of Arch, we have decided to integrate them. To summarise, each question can be attributed to one of the 3 following components: functional core, dialogue and presentation. It is also possible to not relate the question to any component of the arch model.

\subsection{Tool Support DREAM}

DREAM has been designed in order to support all the features of the TEAM notation presented above. It has been designed to support users' activities such as edition of TEAM diagrams (including storing related documents for argumentation), modification and visualization techniques for handling large diagrams. DREAM is available on the web and can be downloaded (together with some excerpts from case studies) at the following address: http://liihs.irit.fr/dream .

During edition phase (corresponding to the recording of information and the exploration of options), DREAM interaction tries to reduce syntax errors (with respect to TEAM syntax) as only authorised relations between two nodes are allowed. DREAM provides a feedback animation when a node is modified. When modification is made on a duplicated node, animation is provided on all entity of this node. This permits user to see the impact of the changes throughout the entire diagram.

DREAM also supports the decision phase (when design decisions are made by designers). 'Classical' QOC diagram can be inconsistent as it is possible to choose an option o1 (appearing in a set of option s1) for a question q1 and but to select a different one 02 (appearing in a different set of option s2 where o1 appears too) for another question q2. This kind of consistency in the choice phase is detected by DREAM and prompted to the user for modification. 


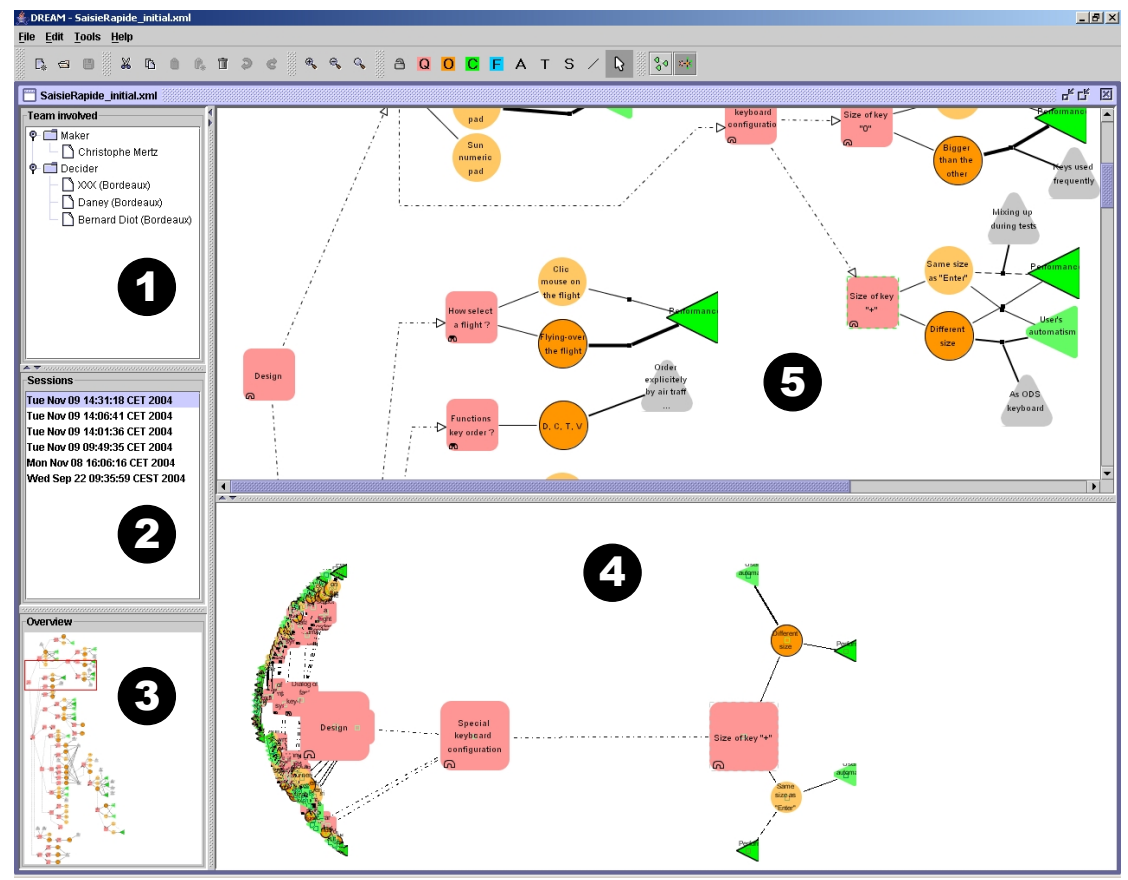

Fig. 3. User Interface of DREAM environment

Usually Design Rationale is seen an element or document only available at the end of the design process. As in User Centred Development the design process is iterative, it is required to support the edition and decision phases in an iterative manner too. We propose to capture each step of the edition of the diagram and to store information about the changes made, who made them and when. The same holds for the decision phase. To support these activities, DREAM tool features a session area (see area 1 and 2 of Figure 3). A session is made of a diagram (and all its related documents), a list of authors, and a date. We distinguish to types of authors involved in the design process. The makers: people who create and elaborate the diagram. The deciders: people who make decisions and give the global trends to follows. Deciders could be customer(s) and/or user(s). Decisions made by makers are mostly guided by deciders. DREAM permits to start new session or to start a new file from a selected session.

One of the critical characteristic of DREAM is that it embeds functionalities that are of great advantages with respect to manual edition of diagrams. One of these is related to the multiple appearances of nodes within a given diagram. In this section we already mentioned this aspect for options but it is also true for criteria, factors, tasks and scenarios. For instance the same factor can appear in several places. Changing the weight of such a factor has an impact on all the other instances of the factor and an animation feedback is provided when a modification is done, to help user to see the impact of the modification on the entire diagram. 
We decided to provide a graphical representation of the each TEAM element that can be interactively manipulated. Each element of the notation is associated with a glyph, a colour, and a label. Label contains a short description of the node, a description more precise can be entered and documents can be attached to any node. Questions are depicted as red square, options as orange disc, criteria as green triangle, factors as blue triangle, and arguments as grey triangle. Glyph and colour, of criteria and factors, evolve according to their weight. A strong weight is represented by a strong glyph (bold border) and brightness colour; whereas a weak weight is represented by a fuzzy glyph and pastel colour.

As stated above, Design Rationale diagrams are expected to be large and containing a large quantity of information. To tackle this, DREAM provides three simultaneous representations that are represented in areas 3, 4 and 5 of Fig. 3. Area 5 is standard visualisation of the diagram that can be zoomed in and zoomed out. This view is dedicated to edition activities. Area 3 displays the entire diagram, and the red rectangle match with the current view in area 5. A third visualisation is bifocal [2] and can be seen in area 4 . This view displays TEAM diagrams in a tree-like way and allows users to focus on detailed part of the tree (right hand part called focus) and to keep a context view of the rest of the tree (left hand part called context+focus). They allow users to focus on one node (a factor for instance) and to see all the nodes that are connected to it. This type of visualization is really useful as it counter balance the problem of allowing multiple appearance of the same node in a diagram.

\section{An Air Traffic Control Case Study}

This section presents the use of TEAM and DREAM on a case study. This case study comes from the Air Traffic Management domain and deals with interaction design issues on an Air Traffic Control workstation.

\subsection{Context}

The DREAM/TEAM approach has been applied to the entire case study and is one of the 3 case studies we developed so far. One of the main objective for this case study were the following:

- Every information concerning design process could be store by means of the TEAM notation;

- Extensions are used and useful with respect to 'classical' QOC;

- DREAM tool supports the various activities of the several kinds of users involved in the design process.

The case study has been developed with colleagues at the CENA (French Centre for Studies on Air Traffic Management) and is based on an interactive application designed and developed by them. The context is the one of 'approach' air traffic controllers i.e. controllers in charge of aircraft approaching an airport. The aircrafts are handed over to them by so called 'enroute' air traffic controllers and they are supposed (after preparing the aircrafts route appropriately) to transfer them to the 'tower' controllers in charge of take-off, landing and taxiing. 
The approach air traffic controller can be in charge of a significant number of aircrafts and might have to issue a lot of clearances ${ }^{1}$ in a very short period of time. This activity is different from the one of the other types of controllers for whom time scale is much longer.

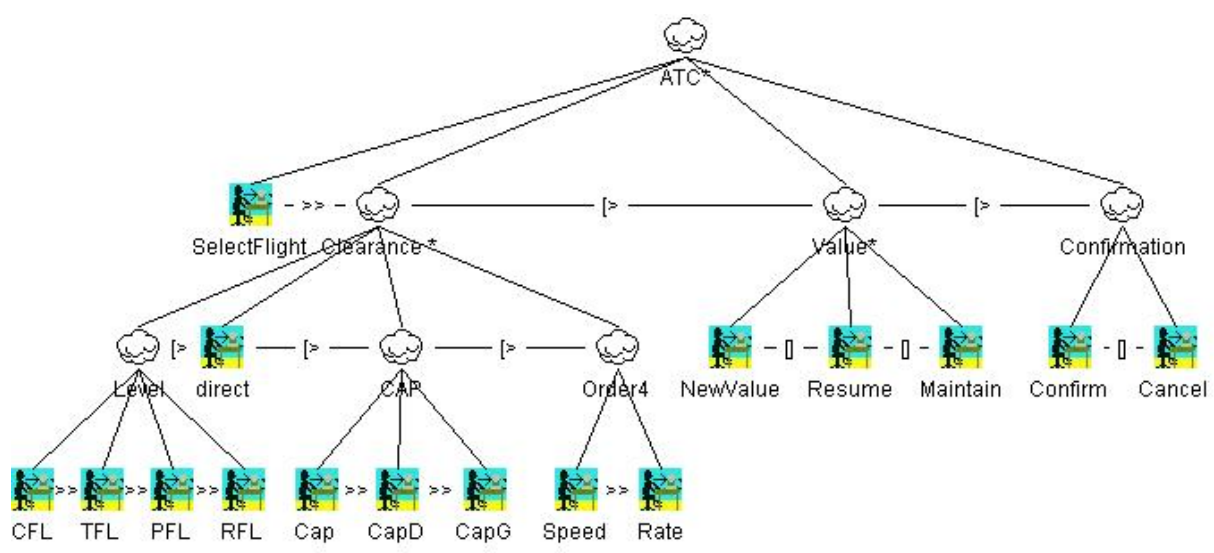

Fig. 4. Task model describing ATC activity

Figure 4 presents (using the CTT notation [24]) a task model of an 'approach' air traffic controller. This task model is only partial but conveys all the information relevant to the case study. The task is divided in four high level sub-tasks: select a flight, select clearance (level, cap (i.e. heading), speed, rate, etc.), select value and confirm or cancel. After selecting the aircraft, the ATC selects the type of clearance to be issued. The selection of the type of clearance is made by pressing at least once on the keys named "level", "cap", "direct" and "speed". The clearance value is typed-in using the keyboard and can be either a new value (i.e. a number), a confirmation "maintain" or a correction "resume" the previous value. A graphical feedback is provided when the value is validated.
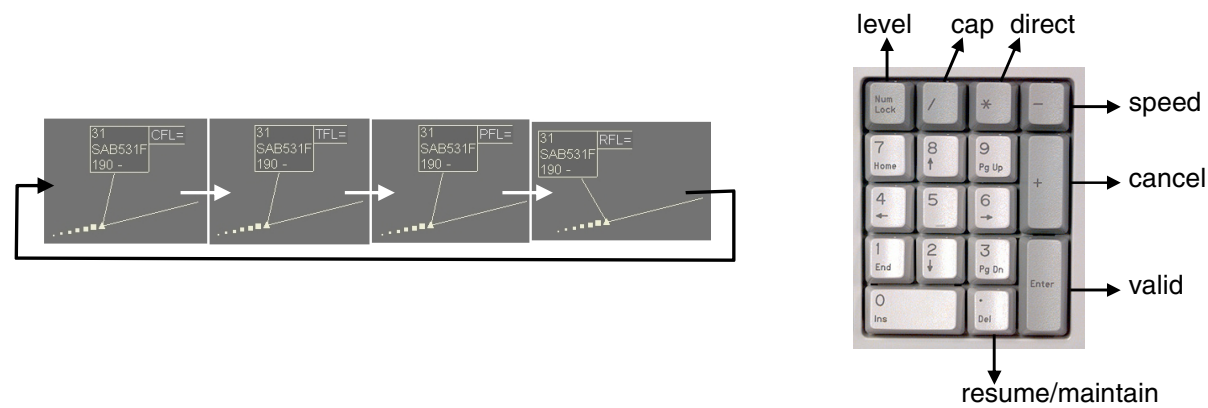

Fig. 5. Left hand part: cyclic access to level menu. Right hand part functions related to keys.

\footnotetext{
${ }^{1}$ A clearance is the name given to the orders sent by the controller to the pilot. This can be changing heading, flight level, speed, ...
} 
Ten kinds of clearances are identified, only four buttons are available. Functions are classified by themes and statically associated to keys (see Fig. 5):

- Key "num lock" corresponds to level menu (CFL, TFL, PFL, RFL);

- Key "/" corresponds to direct menu;

- Key "*" corresponds to cap menu (Cap, Right Cap, Left Cap) ;

- Key "-" corresponds to speed menu (Speed, Rate).

Fig. 5 (right part) presents the four functions accessible sequentially via key "num lock". Menus are cyclic, for example to reach PFL function user press three times (modulo four) key "num lock".

\subsection{Rationalisation}

The interactive system designed by CENA was mainly aimed at allowing ATC to issue clearances as rapidly as possible. Other (secondary) concerns were making error rate as low as possible and avoiding syntactically incorrect clearances as much as possible. The current interactive application consists in a radar screen, a mouse and a keyboard. The radar screen displays all flights controlled by the air traffic controller as well as incoming and outgoing flights. Each flight is graphically represented by a label (see left hand side of Figure 5), a set of dots representing the past 5 positions of the aircraft and speed vector (a straight line) providing information about both speed (line length) and direction (line direction). The flight label contains the following information flight id, plane id and altitude level.

The design team at CENA followed a user centred design [18] and iterative approach. After some information gathering, they implemented a first prototype. This version evolved during several meetings involving users and designers. After each meeting minutes were prepared and distributed. Those meeting, held on a regular basis, offered the opportunity to users to comment and practice the various prototypes. The process ended after the tenth meeting.

In order to validate the DREAM/TEAM approach we used the tool and the notation to model all the information contained in the minutes. The information gathered was ranging from graphical design sketches (representing graphical appearance of objects), automata (describing the interaction technique) as well as decisions about the retained and discarded design options. A last report summarising all the choices made during the meeting was also available and has been used by CENA team as a set of requirements for the development of the final application. In order to trace the process we built diagrams starting from these ten reports and the summarizing report. We built a first diagram and this diagram evolved with information extracted from meetings in a chronological way. Only six sessions appear in the diagram as four sessions were not containing relevant information or ended prematurely.

Customers/users gave two strong requirements: data entries have to be fast, and application had to be quickly implemented.

\subsection{Modelling with TEAM}

For space reasons we do not present the entire models resulting from the design rationale activity. A more complete one can be found in [25] this section the modelling process itself but we describe the output of the process. Task models and scenarios 
were not used in the design process by CENA and this is the reason why do not appear on the diagram. Four criteria were identified and are duplicated (i.e. "fast", "honesty", "user's automatism", and "efficient quickly"). They are connected to most of the options. The resulting diagram is displayed on Fig. 6 and contains 203 nodes (41 questions, 55 options, 21 criteria, 6 factors, and 20 arguments). The point here is not to describe individually each node but to present salient information that provides insights about the process, the notation and the tool.

Left hand side of Fig. 6 presents a subset of the entire diagram resulting from the modelling process while right hand side emphasises one frame extracted from the diagram that is discussed hereafter.

The five rounded rectangles highlight one couple criterion/factor. This couple appears five times in the diagram. The criterion is "performance" meaning that performance can be assessed in several places in the diagram for several options. The factor is "fast" and models a customer/user requirement that interaction is required to be fast.

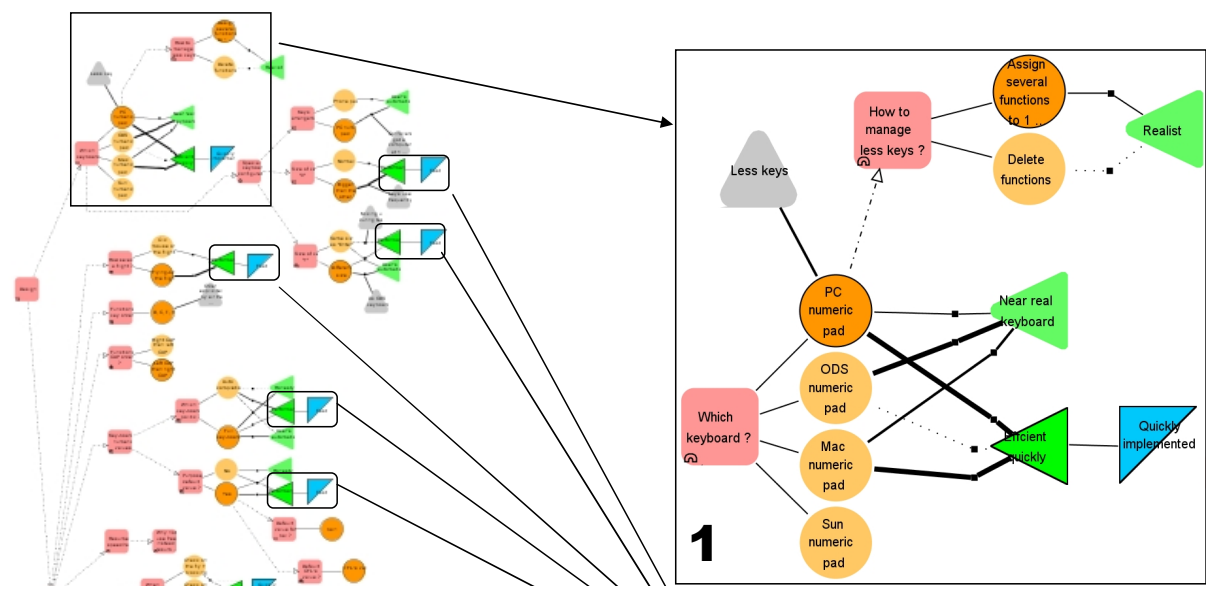

Fig. 6. Excerpt of the entire diagram of the case study

Frame (enlarged on the right hand side of Fig. 6) deals with the question of choice of keyboard, options considered are PC numeric pad, ODS numeric pad (keyboard already available on ATC workstations), Macintosh numeric pad, and Sun numeric pad. Options are valuated (expected Sun num pad option) by two criteria: "near real keyboard" and "efficient quickly". Intuitively, we would suppose that designers would select ODS numeric pad option but in fact they chose PC numeric pad instead. One of the main reasons for this choice lies in the other factor "easy to implement" and this get a strong weight. Besides, as modelled, key arrangement of the PC numeric pad is closer to the ODS one keyboard. However, as shown in frame number one this design choice raised a new question: "how to manage fewer keys?" i.e. how to have as few keys as possible for entering clearances.

Graphical visualisation supports the analysis of diagrams in order to detect questions poorly investigated or design choices under discussion. Indeed, it is easy to spot 
(even on a large diagram) a question related to only one option and (2) options are not related to any criterion.

During the six meeting providing relevant data, diagrams evolved. Some parts changed often as, for instance the part concerning syntax for input of clearances' values. DREAM versioning facilities provide an easy way to support this kind of activity.

\subsection{Rationalising DREAM}

Diagram is Fig. 7 shows the design rationale result about the design of the graphical representation of TEAM diagrams in DREAM. This design of this graphical representation has been done with Jean-Luc Vinot graphical designer. From left to right we can see the design questions (how to represent each node in TEAM) and then the set of options that have been envisaged. Criteria are related to the tasks of editing (e.g. easiness to distinguish the various types of nodes), some effort constraints (e.g. easiness to implement) and some perception considerations (e.g. reading by block, perceptive colour, perceptive brightness ...). The main point of this task was to assess whether TEAM notation was able to capture such graphical rationale that is outside the initial scope of the notation and the tool.

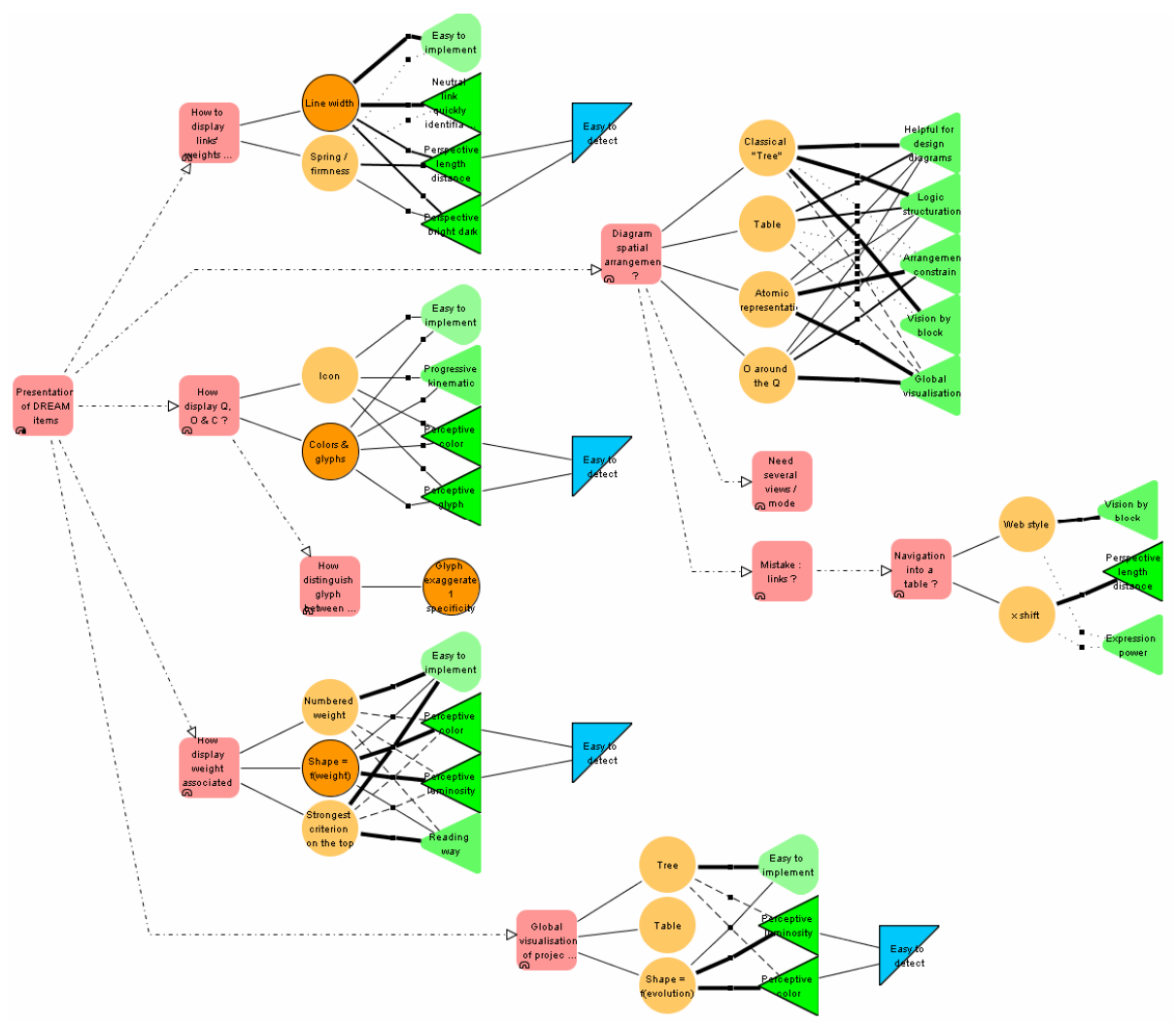

Fig. 7. Rationalisation of the graphical representation of DREAM notation 


\section{Conclusion}

In this paper we have presented a notation (TEAM) and its case tool (DREAM) that are dedicated to the engineering of interactive systems. The approach presented here focuses on the design process and more precisely on the traceability and rationalisation. The notation is anchored in HCI research and provides explicit links to architecture (Arch), user centred development process (iterative prototyping), task analysis and modelling and scenarios and thus provides a generic framework for gathering these multiple information sources in a single model.

The approach builds on existing work in the field of design rationale integrating successful aspects and providing solutions to the identified limitations. The approach has been applied to several case studies including interaction techniques design, graphic design and software engineering side of interactive systems design.

\section{Acknowledgement}

We would like to thank members of the former CENA (Research Centre on Air Traffic Control in Toulouse) namely Christophe Mertz who developed the ATC system presented in this case study and Jean-Luc Vinot (graphic designer at CENA) who designed the graphical representation of TEAM and accepted to rationalise the process.

\section{References}

1. Bass, L., Little, R., Pellegrino, R., Reed, S., Seacord, R., Sheppard, S., Szezur, M.: The Arch Model: Seeheim Revisited. In: User Interface Developpers' Workshop (1991)

2. Cava, R.A., Luzzardi, P.R.G., Freitas, C.M.D.S.: The Bifocal Tree: a Technique for the Visualization of Hierarchical Information Structures. In: IHC 2002 - 5th Workshop On Human Factors In Computer Systems, Fortaleza (2002)

3. Compendium is available at: http:www.compendiuminstitute.org/tools/compendium.htm

4. Conklin, J., Begeman, M.L.: GIBIS: A Hypertext Tool for Exploratory Policy Discussion. ACM Transactions on Office Information Systems 6(4), 303-331 (1988)

5. Conklin, J., Burgess-Yakemovic, K.C.: A Process-Oriented Approach to Design Rationale. In: [17], pp. 393-427 (1996)

6. ESARR 6. EUROCONTROL Safety Regulatory Requirement. Software in ATM Systems. Edition 1.0 (2003), http://www.eurocontrol.int/src/public/standard_page/esarr6.html

7. Farenc, C., Palanque, P.: Exploitation Des Notations De Design Rationale Pour Une Conception Justifiée Des Applications Interactives. IHM'99. 11ièmes Journées Sur L'Ingénierie Des Interfaces Homme-Machine (1999)

8. Green, M.: Report on Dialogue Specification Tools. User Interface Management Systems, pp. 9-20. Springer, Heidelberg (1985)

9. Johnson, C.W.: Using Design Rationale To Support Formal Methods In The Development of Human-Computer Interfaces. HCI Journal 11(4), 291-320 (1996)

10. Kunz, W., Rittel, H.: Issues As Elements of Information Systems. PhD, Univ. of California (1970) 
11. Lacaze, X., Palanque, P., Navarre, D.: Evaluation de Performance et Modèles de Tâches Comme Support a la Conception Rationnelle des Systèmes Interactifs. 14eme Conférence Francophone sur L'Interaction Homme Machine. In: IHM 2002, pp. 17-24 (2002)

12. Lee, J.: SIBYL: A Tool for Managing Group Design Rationale. Computer Supported Cooperative Work, pp. 79-92. ACM Press, New York (1990)

13. Lee, J.: Extending the Potts and Bruns Model for Recording Design Rationale. In: Proceedings of the 13th International Conference on Software Engineering, pp. 114-125 (1991)

14. Lee, J., Laï.: What's in Design Rationale. In: [17], pp. 21-51 (1996)

15. MacLean, A., Young, R.M., Bellotti, V.M.E., Moran, T.P.: Questions, Options, Criteria: Elements of Design Space Analysis. In: [17] (1996)

16. McCall, J., Richards, P., Walters, G.: Factors in Software Quality. Rome Air Development Center (RADC), RADC-TR-77-369, vol. III (November 1977)

17. Moran, T.P., Carroll, J.M.(eds.): Design Rationale: Concepts, Techniques, and Use. Lawrence Erlbaum Associates, Mahwah (1996)

18. Norman, D.A., Draper, S.W.: User-Centred System Design: New Perspectives on Human Computer Interaction. Lawrence Erlbaum Associates, Hillsdale (1986)

19. Ormerod, T.C., Mariani, J., Ball, L.J., Lambell, N.: Desperado: Three-in-one indexing for innovative design: Interact -Seventh IFIP Conference on Human-Computer Interaction. IOS Press, London (1999)

20. Potts, C., Bruns, G.: Recording the Reasons for Design Decisions. In: Proceedings of the 10th International Conference on Software Engineering, pp. 418-427 (1988)

21. RTCA/DO-178B. Software Considerations in Airborne Systems and Equipment Certification (December 1, 1992), http://www.rtca.org/

22. Sage, M., Johnson, C.W.: Pragmatic Formal Design: A Case Study in Integrating Formal Methods into the HCI Development Cycle. In: DSVIS, pp. 134-155. Springer, Heidelberg (1998)

23. Shum, S.J.: Cognitive analysis of design rationale representation. PhD Thesis. York, UK: Department of Psychology, University of York (1991).

24. Paternò, F.: Model-Based Design and Evaluation of Interactive Application. Springer, Heidelberg (1999)

25. Lacaze, X., Palanque, P., Barboni, E., Bastide, R., Navarre, D.: From DREAM to Realitiy: Specificities of Interactive Systems Development with respect to Rationale Management. In: Dutoit, A.H., McCall, R., Mistrik, I., Paech, B. (eds.) Rationale Management in Software Engineering, pp. 155-172. Springer, Heidelberg (2006) 